\title{
Warm-polytropic inflationary universe model
}

\author{
M. R. Setare ${ }^{a 1}$, M. J. S. Houndjo ${ }^{b 2}$, V. Kamali ${ }^{a 3}$ \\ a Department of Science, Campus of Bijar, University of Kurdistan \\ Bijar, IRAN \\ ${ }^{b}$ Institut de Mathématiques et de Sciences Physiques (IMSP) \\ 01 BP 613 Porto-Novo, Bénin
}

\begin{abstract}
In the present paper we study warm inflationary universe models in the context of a polytropic gas. We derive the characteristics of this model in slow-roll approximation and develop our model in two cases, 1- For a constant dissipative parameter $\Gamma$. 2- $\Gamma$ as a function of scalar field $\phi$. In these cases we will obtain exact solution for the scalar field and Hubble parameter. We will also obtain explicit expressions for the tensor-scalar ratio $R$, scalar spectrum index $n_{s}$ and its running $\alpha_{s}$, in slow-roll approximation.
\end{abstract}

Keywords: inflation; polytropic; slow-roll approximation; tensor-scalar ratio.

PACS Number(s): $98.80 . \mathrm{Cq}$

\footnotetext{
${ }^{1}$ E-mail: rezakord@ipm.ir

${ }^{2}$ E-mail: sthoundjo@yahoo.fr

${ }^{3}$ E-mail: vkamali1362@gmail.com
} 


\section{INTRODUCTION}

As is well known, evidence from the cosmic microwave background radiation indicates the early universe underwent an accelerating phase i.e, the inflationary epoch. Moreover an inflationary phase is one of the most compelling solution to many long-standing problems of the standard hot big bang scenario, for example, the flatness, the horizon, and the monopole problems, among others [1, 2]. Scalar field as a source of inflation provides the causal interpretation of the origin of the distribution of large scale structure and observed anisotropy of cosmological microwave background (CMB) [3, 4]. In standard models for inflationary universe, the inflation period is divided into two regimes, slow-roll and reheating epochs. In slow-roll period kinetic energy remains small compared to the potential terms. In this period, all interactions between scalar fields (inflatons) and other fields are neglected and the universe inflates. Subsequently, in reheating period, the kinetic energy is comparable to the potential energy and inflaton starts an oscillation around the minimum of the potential losing their energy to other fields present in the theory. So, the reheating is the end period of inflation.

In warm inflationary models radiation production occurs during inflationary period and reheating is avoided [5, 6]. Thermal fluctuations may be obtained during warm inflation. These fluctuations could play a dominant role to produce initial fluctuations which are necessary for Large-Scale Structure (LSS) formation. So the density fluctuation arises from thermal rather than quantum fluctuation [7-10]. Warm inflationary period ends when the universe stops inflating. After this period the universe enters in radiation phase smoothly $[1,2]$. Finally, remaining inflatons or dominant radiation fields created the matter components of the universe.

On the other hand, the polytropic gas has been proposed as an alternative model for describing the accelerating of the universe [11]. Polytropic equation of state has been used in various astrophysical situations, for example it can explain the equation of state of degenerate white dwarfs, neutron stars and also the equation of state of main sequence stars [12], and in the case of Lane-Emden models 13, 14]. The equation of state of polytropic model may be seen in mathematical biology [15], statistical physics [16] and astronomy [17, 18]. This model may be useful in cosmology [19 21]. In Ref.[19] the simple models of our universe have been studied using a generalized equation of state $P=\left(\alpha+k \rho^{\frac{1}{n}}\right) \rho$. This model have two 
components in equation of state: 1- A linear component $P=\alpha \rho$. 2- Polytropic component $P=k \rho^{1+\frac{1}{n}}$. The early universe without singularity may be described by this generalized equation of state where $\alpha=\frac{1}{3}, n=1, k=-\frac{4}{3 \rho_{p}}$ and Planck density $\rho_{p}=5.16 \times 10^{99} \frac{\mathrm{gr}}{\mathrm{m}^{3}}$. For a given generalized polytropic equation of state the inflationary expansion is driven by using vacuum energy [19]. The inflation epoch happened on a time-scale of a few Planck times $t_{p}=5.39 \times 10^{-44}$ and the universe brings to a size $a_{1}=1.611 \times 10^{-6} \mathrm{~m}$. After this period $\left(t \gg t_{p}\right)$ the universe inters in radiation era. In pre-radiation era $\left(a \ll a_{1}\right)$, it was shown that $\rho \simeq \rho_{p}$ where the Planck density $\rho_{p}$ can represent an upper bound for density [19]. For a constant value of density we have a phase of early inflation. In the present work we initiate warm-inflation model by using the polytropic equation of state, where the inflaton fields have an interaction with the radiation field.

Previously chaotic inflation in the context a phenomenological modification of gravity inspired by the Chaplygin gas equation of state has been studied by Bertolami et al [22] According to this model, the scalar field, which drives inflation, is the standard inflaton field, can be extrapolate for obtaining a successful inflation period with a Chaplygin gas model. After that this scenario extended to the Chaplygin inspired inflationary model in which a brane-world model is considered [23]. In the paper [24], del Campo et al have studied Warm inflationary universe models in the context of a Chaplygin gas equation.

Based on the above statements we are motivated to consider early universe cosmological implications of this model and investigate if it can inspire warm inflation like the model of $[24]$.

\section{THE MODEL}

The polytropic fluid has been proposed as an alternative model for describing the accelerating of the universe where its equation of state is of the form

$$
p=K \rho^{1+\frac{1}{n}}
$$

where $K$ and $n$ are constant values called in the literature polytropic constant and polytropic index, respectively. The polytropic constant $K$ can take the positive value for radiation and stiff fluid, the zero value for dust and the negative value for inflationary scenario [11]. From 
equation of state (11) we can find squared speed of polytropic fluid as

$$
v_{g}^{2}=\frac{d p}{d \rho}=K\left(1+\frac{1}{n}\right) \rho^{\frac{1}{n}}
$$

so this fluid is stable where [25, 26]

$$
v_{g}^{2}>0 \Rightarrow \quad K<0,-1<n<0 \quad \text { and } \quad K>0, n>0, n<-1
$$

The $n=-\frac{1}{2}$ case is motivated by string theory [27-32]. We have the conservation equation as

$$
\dot{\rho}+3 H(\rho+p)=0
$$

where dots mean derivatives with respect to the cosmological time. Now, using (11) and (4) we can obtain

$$
\rho_{p t}=\left[-K+B a^{\frac{3}{n}}\right]^{-n}
$$

where $a(t)$ is scale factor and $B$ is positive integration constant. In this article we will not consider the above equation as a consequence of the polytropic equation of state (11), but we start by studying the modified gravity, where the gravitational dynamic is given by modified Friedmann equation as

$$
H^{2}=\kappa\left(\left[-K+\rho_{\phi}^{-\frac{1}{n}}\right]^{-n}+\rho_{\gamma}\right)
$$

where $H=\dot{a} / a$ is the Hubble parameter. The $\rho_{\gamma}$ is the radiation energy density. We also assume that $\kappa=8 \pi G / 3=8 \pi /\left(3 m_{p}^{2}\right)$ ( $m_{p}$ is Planck mass) and $\rho_{\phi}=\frac{\dot{\phi}}{2}+V(\phi)(V(\phi)$ is scalar potential). This modification of energy density is understood from an extrapolation of equation (5) as

$$
\rho_{p t}=\left[-K+\rho_{m}^{-\frac{1}{n}}\right]^{-n} \rightarrow\left[-K+\rho_{\phi}^{-\frac{1}{n}}\right]
$$

where $\rho_{m}$ is the matter energy density [22]. The dynamics of the cosmological model in the warm polytropic inflationary scenario is given by

$$
\ddot{\phi}+3 H \dot{\phi}+V^{\prime}=-\Gamma \dot{\phi}
$$

and

$$
\dot{\rho}_{\gamma}+4 H \rho_{\gamma}=\Gamma \dot{\phi}^{2}
$$

where $\Gamma$ is the dissipation coefficient, which describe the decay of the scalar field into radiation during the inflation. The pressure of a scalar field is expressed as

$$
p_{\phi}=\frac{1}{2} \dot{\phi}^{2}-V(\phi)
$$


Assuming the set of slow-roll conditions, i.e. $\dot{\phi}^{2} \ll V(\phi)$ and $\ddot{\phi} \ll(3 H+\Gamma) \dot{\phi}$, the Friedmann equation (7) reduces to

$$
H^{2} \simeq \kappa\left[-K+V^{\frac{-1}{n}}\right]^{-n}
$$

also equation (8) reduces to

$$
3 H(1+r) \dot{\phi} \simeq-V^{\prime}
$$

where

$$
r=\frac{\Gamma}{3 H}
$$

By considering that during warm inflation the radiation production is quasi-stable, i.e $\dot{\rho}_{\gamma} \ll$ $4 H \rho_{\gamma}$ and $\dot{\rho_{\gamma}} \ll \Gamma \dot{\phi}^{2}$, from Eq.(9) we obtain

$$
\rho_{\gamma}=\frac{\Gamma \dot{\phi}^{2}}{4 H} .
$$

Using the Stefan-Boltzmann formula, we have $\rho_{\gamma}=\sigma T_{r}^{4}$, where $\sigma$ is the Stefan-Boltzmann constant, and $T_{r}$ is the temperature of radiation. Using Eqs.(12),(13),(14), we obtain

$$
\rho_{\gamma}=\sigma T_{r}^{4}=\frac{r V^{\prime 2}}{12(1+r)^{2} \kappa}\left[-K+V^{\frac{-1}{n}}\right]^{n}
$$

Introducing the dimensionless slow-roll parameters, we can write

$$
\varepsilon=-\frac{\dot{H}}{H^{2}} \simeq \frac{V^{\prime 2}}{6 \kappa(1+r)} V^{\frac{-1}{n}-1}\left[-K+V^{\frac{-1}{n}}\right]^{n-1}
$$

Also,

$$
\eta \equiv \frac{-\ddot{H}}{H \dot{H}}=\frac{\left[-K+V^{\frac{-1}{n}}\right]^{n}}{3 \kappa(1+r)}\left[V^{\prime \prime}+\frac{V^{\prime 2}}{V}+\frac{V^{\prime 2} V^{\frac{-1}{n}-1}}{n\left(-K+V^{\frac{-1}{n}}\right)}\right]
$$

Using Eqs.(12), (15) we find following relation between $\rho_{\gamma}$ and $\rho_{\phi}$

$$
\rho_{\gamma}=\frac{r \varepsilon}{2(1+r)} \rho_{\phi}^{\frac{1}{n}+1}\left(-K+\rho_{\phi}^{\frac{-1}{n}}\right)
$$

The condition under which inflation takes place can be summarized with the parameter $\varepsilon$ satisfying the inequality $\varepsilon<1$, which is analogue to the requirement that $\ddot{a}>0$. This condition could be written as

$$
\rho_{\phi}^{\frac{1}{n}+1}\left(-K+\rho_{\phi}^{\frac{-1}{n}}\right)>\frac{2(1+r)}{r} \rho_{\gamma}
$$

Inflation ends when the universe heats up at a time when $\varepsilon \simeq 1$, which implies

$$
\rho_{\phi}^{\frac{1}{n}+1}\left(-K+\rho_{\phi}^{\frac{-1}{n}}\right) \simeq \frac{2(1+r)}{r} \rho_{\gamma}
$$

The number of e-folds at the end of inflation is given by

$$
N=\int H d t=\int \frac{H}{\dot{\phi}} d \phi=-3 \kappa \int_{\phi_{*}}^{\phi_{f}} \frac{\left[-K+V^{\frac{-1}{n}}\right]^{-n}}{V^{\prime}}(1+r) d \phi
$$




\section{PERTURBATIONS}

In this section we will study the perturbations for our model. In quantum cosmology the interesting primary quantity is the curvature perturbation spectrum which can be extract from correlation of two quantum fields in the same time. The spectrum is a measure of size of field fluctuations. The density of perturbation $\delta_{H}$ in term of quantum fluctuation $\delta \phi$ is given by

$$
\delta_{H} \sim \delta N \sim H \delta t \sim H \frac{\delta t}{\delta \phi} \delta \phi \sim \frac{H}{\dot{\phi}} \delta \phi
$$

From Refs. [5, 33 35] the density perturbation may be written as

$$
\delta_{H}=\frac{2 H}{5 \dot{\phi}} \delta \phi
$$

Using Eqs.(7) and (8) we can write

$$
\delta_{H}^{2}=\frac{36(1+r)^{2}}{25 V^{\prime 2}} \kappa^{2}\left[-K+V^{\frac{-1}{n}}\right]^{-2 n} \delta \phi^{2}
$$

when dissipation is large, the dissipation coefficient $\Gamma$ is much larger than the rate expansion $H$, so $r=\frac{\Gamma}{3 H} \gg 1$. In warm inflationary scenario, the fluctuations of scalar field $\delta \phi$ are not generated by quantum fluctuations but the fluctuations are generated by thermal interaction with the radiation fields, therefore as have been shown by Taylor and Berera [36], we have

$$
(\delta \phi)^{2} \simeq \frac{k_{F} T_{r}}{2 \pi^{2}}
$$

where $k_{F}$ is wave-number and given by $k_{F}=H \sqrt{3 r}$. Now using Eq.(25) we can rewrite Eq.(24) as following

$$
\delta_{H}^{2} \simeq \frac{18 \sqrt{3}}{25 \pi^{2} V^{\prime 2}}\left[\kappa r\left(-K+V^{\frac{-1}{n}}\right)^{-n}\right]^{\frac{5}{2}}=\frac{36 \sqrt{3} r^{\frac{5}{2}} \kappa^{2} T_{r}}{2 \pi^{2} V^{\prime 2}}\left[-K+V^{-\frac{1}{n}}\right]^{-3 n}
$$

We find spectral index $n_{s}\left(\right.$ where $\left.n_{s}-1=\frac{d \ln \delta_{H}^{2}}{d \ln k}\right)$

$$
n_{s} \approx 1+2 \tilde{\eta}-5 \tilde{\epsilon}+\zeta
$$

where

$$
\begin{gathered}
\tilde{\epsilon}=\frac{V^{\prime 2} V^{-\frac{1}{n}-1}}{6 \kappa r}\left[-K+V^{-\frac{1}{n}}\right]^{n-1} \\
\tilde{\eta}=\frac{\left[-K+V^{-\frac{1}{n}}\right]^{n}}{3 \kappa r}\left(V^{\prime \prime}+\frac{V^{\prime 2}}{V}+\frac{V^{\prime 2} V^{-\frac{1}{n}-1}}{n\left(-K+V^{-\frac{1}{n}}\right)}\right)
\end{gathered}
$$


and

$$
\zeta=-\frac{2 V^{\prime}\left[-K+V^{-\frac{1}{n}}\right]^{n}}{3 \kappa r}\left(\frac{V^{\prime}}{V}+\frac{5 r^{\prime}}{4 r}+\frac{V^{\prime} V^{-\frac{1}{n}-1}}{n\left[-K+V^{-\frac{1}{n}}\right]}\right)
$$

In the above equation we have used the relation between number of e-folds and interval in wave-number $d \ln k=-d N$. Running of the scalar spectral index

$$
\alpha_{s}=\frac{d n_{s}}{d \ln k}=\frac{2 V^{\frac{1}{n}+1}}{V^{\prime}}\left[-K+V^{-\frac{1}{n}}\right] \tilde{\epsilon}\left(-5 \tilde{\epsilon}^{\prime}+2 \tilde{\eta}^{\prime}+\zeta^{\prime}\right)
$$

is one of the interesting parameters which is obtained from data of WMAP observations. From WMAP7 results, $\alpha_{s}$ is approximately -0.038 [37]. Power spectrum of the tensor perturbation during inflation epoch is given by following relation

$$
A_{g}^{2}=6 \kappa\left(\frac{H}{2 \pi}\right)^{2} \operatorname{coth}\left[\frac{k}{2 T}\right]=\frac{3 k^{2}}{2 \pi^{2}}\left[-K+V^{-\frac{1}{n}}\right]^{-n} \operatorname{coth}\left[\frac{k}{2 T}\right]
$$

where $T$ in extra factor $\operatorname{coth}\left[\frac{k}{2 T}\right]$ denotes the temperature of the thermal background of gravitational waves [38]. From Ref. [38] we have, $A_{g}^{2} \propto k^{n_{g}} \operatorname{coth}(k / 2 T)$, so spectral index $n_{g}$ is obtained from power spectrum $A_{g}$ as

$$
n_{g}=\frac{d}{d \ln k} d \ln \left[\frac{A_{g}^{2}}{\operatorname{coth}[k / 2 T]}\right]=-2 \epsilon
$$

Using Eqs.(26) and (32), for high dissipative regime $(\Gamma>3 H)$, tensor-scalar ratio may be written as

$$
R(k)=\left.\left(\frac{A_{g}^{2}}{\mathcal{P}_{R}}\right)\right|_{k=k_{*}}=\left.\frac{V^{\prime 2}\left[-K+V^{-\frac{1}{n}}\right]^{2 n}}{\sqrt{3} T_{r} r^{5 / 2}} \operatorname{coth}\left[\frac{k}{2 T}\right]\right|_{k=k_{*}}
$$

where $\mathcal{P}_{R}=25 \delta_{H}^{2} / 4$. An upper bound for parameter $R$ is obtained from observation data, $\left(R\left(k_{*}=0.002 \mathrm{Mp}^{-1}\right)<0.36\right)[39]$. In next section we would like to consider our model in two important choices for dissipative parameter $\Gamma$. $1-\Gamma$ is a constant parameter, $2-\Gamma$ as a function of scalar field $\phi$.

\section{CHAOTIC INFLATION}

In this section we will consider scalar warm-polytropic inflation model with chaotic potential $V=\frac{m^{2} \phi^{2}}{2}$ (where $m$ is the mass of inflaton) and in high dissipative limit. If the inflaton $\phi$ has the polynomial interactions with the bath environment, one could take the polynomial of $\phi$ for dissipation parameter $\Gamma\left(\Gamma=\Gamma_{p} \phi^{p}\right)$ [40]. The dissipation coefficient 
must be positive, therefor $\Gamma_{p}>0$. As argued in Ref.[40], if the potential $V(\phi)$ is invariant under the transformation $\phi \rightarrow-\phi$, the index $p$ may be zero or even integer. $p=1,2$ cases have been considered in Refs. [7, 41, 42] and general case for $p$ have been studied in Refs. [24, 40, 43]. We will study this model for constant parameter $\Gamma=\Gamma_{0}$ and variable dissipation parameter $\Gamma=\alpha_{p} \phi^{p}$.

\section{A. $\Gamma=\Gamma_{0}=$ const}

Using Eq.(12) and chaotic potential, we get

$$
\dot{\phi}=-\frac{m^{2}}{\Gamma_{0}} \phi \Rightarrow \phi=\phi_{0} \exp \left(-\frac{m^{2} t}{\Gamma_{0}}\right)
$$

From above equation and Eq.(11) Hubble parameter is obtained

$$
H=\sqrt{\kappa}\left[-K+\left(\frac{1}{2} m^{2} \phi_{0}^{2} e^{-2 m^{2} t / \Gamma_{0}}\right)^{-\frac{1}{n}}\right]^{-\frac{n}{2}}
$$

We obtain dissipation parameter $r$ from above equation and Eq.(13)

$$
r=\frac{\Gamma_{0}}{3 \sqrt{\kappa}}\left[-K+\left(\frac{1}{2} m^{2} \phi_{0}^{2} e^{-2 m^{2} t / \Gamma_{0}}\right)^{-\frac{1}{n}}\right]^{\frac{n}{2}}
$$

Energy density of the radiation field is related to the energy density of the inflaton as

$$
\rho_{\gamma}=\frac{\Gamma \dot{\phi}^{2}}{4 H}=\frac{m^{2} \rho_{\phi}}{2 \sqrt{\kappa} \Gamma_{0}}\left[-K+\rho_{\phi}^{-\frac{1}{n}}\right]^{\frac{n}{2}}
$$

By using Eq.(26) scalar power spectrum becomes

$$
\mathcal{P}_{R}=\frac{\Gamma_{0}^{2} \sqrt{\Gamma_{0}} T_{r}}{4 \pi^{2} m^{2} \sqrt{\kappa} V}\left[-K+V^{-\frac{1}{n}}\right]^{-\frac{7 n}{4}}
$$

Tensor-scalar ratio is obtained from Eq.(34)

$$
R(k)=\frac{18 m^{2} V}{T_{r}}\left(\frac{\sqrt{\kappa}}{\Gamma_{0}}\right)^{\frac{5}{2}}\left[-K+V^{-\frac{1}{n}}\right]^{\frac{3 n}{4}} \operatorname{coth}\left[\frac{k}{2 T}\right]
$$

From WMAP7 results $\left(P_{R} \simeq 2.28 \times 10^{-9}, r=0.017<0.36\right)$ and Eqs.(39), (40) $V_{*}$ and $K$ become

$$
V_{*}=2.6 \times 10^{-11} \frac{T_{r}}{m^{2}} \frac{\Gamma_{0}^{\frac{5}{2}}}{\kappa^{29 / 16} \operatorname{coth}\left(\frac{k}{2 T}\right)}
$$

and

$$
K=V_{*}^{-\frac{1}{n}}-\left(\frac{\Gamma_{0}^{5 / 2} T_{r}}{4 \pi^{2} m^{2} \sqrt{\kappa} V_{*}}\right)^{\frac{4}{7 n}}
$$


In the above equation we have $T \simeq T_{r}$. If we choose $K<0$, from above equations, we will get an upper limit for $m$

$$
m^{2}<T_{r} \frac{\Gamma_{0}^{\frac{5}{2}}}{\kappa^{119 / 112} \operatorname{coth}^{3 / 4}\left(\frac{k}{2 T}\right)} 2.1 \times 10^{-5}
$$

and a lower limit for chaotic potential

$$
V_{*}>\frac{\kappa^{-\frac{3}{4}}}{\operatorname{coth}\left(\frac{k}{2 T}\right)} \times 10^{-6}
$$

Now, using forthcoming Planck data $(R \geq 0.01)$ [44, 45], from Eqs. (39) and (40) we derive

$$
V_{*}=1.8 \times 10^{-13} \frac{T_{r}}{m^{2}} \frac{\Gamma_{0}^{\frac{5}{2}}}{\kappa^{29 / 16} \operatorname{coth}\left(\frac{k}{2 T}\right)}
$$

When $K<0$, we find

$$
m^{2}<2.7 \times 10^{-10} T_{r} \frac{\Gamma_{0}^{\frac{5}{2}}}{\kappa^{119 / 112} \operatorname{coth}^{3 / 4}\left(\frac{k}{2 T}\right)}
$$

We have found further constrain on the mass of Chaotic potential, from forthcoming Planck data. In this article we have considered the stable polytropic fluid where $K<0$ and $-1<n<0$, from equation (39) and (40) we find

$$
-K+V^{-\frac{1}{n}}>0
$$

So using the above equation and Eq.(44) we obtain following constraint on the value $n$

$$
n=\frac{1}{2 q} \quad q<0
$$

Therefore, in warm-polytropic inflation model with chaotic potential, we have obtained an extra constrain (48) on the parameter $n$. By using Eqs.(27),(41),(42) we have plotted parameter $m^{2}$ versus the dissipation parameter $\Gamma_{0}$ in Fig.(1).

\section{B. $\Gamma=\Gamma(\phi)$ case}

Now we consider a situation with a power-law dissipation coefficient

$$
\Gamma=\alpha_{p} \phi^{p}
$$


where $p$ is positive and integer. By using Eqs.(12) and (49) scalar field is obtained

$$
\phi(t)=\left[\phi_{i}^{p}-\frac{p m^{2} t}{\alpha_{p}}\right]^{\frac{1}{p}} \quad \phi(t=0)=\phi_{i}
$$

We find dissipative parameter $r$ in term of cosmological time

$$
r(t)=\frac{\alpha_{p}}{3 \sqrt{\kappa}}\left(\phi_{i}^{p}-\frac{p m^{2} t}{\alpha_{p}}\right)\left[-K+\left(\frac{1}{2} m^{2} \phi^{2}\right)^{-\frac{1}{n}}\right]^{-\frac{1}{2 n}}
$$

In high dissipative regime $(r \gg 1)$ we have

$$
\frac{\alpha_{p}}{3 \sqrt{\kappa}}\left(\phi_{i}^{p}-\frac{p m^{2} t}{\alpha_{p}}\right) \gg\left(-K+\left(\frac{1}{2} m^{2} \phi^{2}\right)^{-\frac{1}{n}}\right)^{\frac{1}{2 n}}
$$

From Eq.(18) we may find a relation between energy densities of radiation field and inflaton field.

$$
\rho_{\gamma}=\frac{m^{p+2}}{\sqrt{\kappa} \alpha_{p} 2^{p / 2+1}} \frac{\rho_{\phi}^{1-\frac{p}{2}}}{\left(-K+\rho_{\phi}^{-\frac{1}{n}}\right)^{-\frac{1}{n}}}
$$

Using Eqs.(26) and (34), scalar power-spectrum and tensor-scalar ratio are given by

$$
\begin{gathered}
\mathcal{P}_{R}=\frac{9 \sqrt{3} \kappa^{\frac{3}{4}}}{4 \pi^{2} m^{\frac{5 p+4}{4}}} \frac{T_{r}}{V^{\frac{4-5 p}{4}}}\left(-K+V^{-\frac{1}{n}}\right)^{\frac{5-12 n^{2}}{4 n}} \\
R(k)=\frac{2 m^{2}\left(3 \sqrt{\kappa} m^{p}\right)^{\frac{5}{2}}}{\left(\sqrt{3} 2^{p} \alpha_{p}\right)^{\frac{5}{2}}} \frac{V^{\frac{4-5 p}{4}}}{T_{r}}\left[-K+\left.V^{\left.-\frac{1}{n}\right]^{\frac{-5+4 n^{2}}{4 n}}} \operatorname{coth}\left[\frac{k}{2 T}\right]\right|_{k=k_{*}}\right.
\end{gathered}
$$

respectively. By using WMAP7 data $\left(P_{R} \simeq 2.28 \times 10^{-9}, r=0.017<0.36\right)$ and from Eqs.(54), (55) the mass of scalar field takes the form (where $T=T_{r}$ )

$$
m=\frac{V^{\beta}}{a}
$$

where

$$
\begin{aligned}
& \beta=\frac{(4-5 p) 8 n^{2}}{(5 p+4)\left(-20 n^{2}+5\right)} \\
& a=\left[\left(\frac{5.7 \times 10^{-9}}{\kappa^{3 / 4} T_{r}}\right)^{4 n^{2}-5}\left(\frac{465 \kappa^{5 / 4} \operatorname{coth}(k / 2 T)}{\left(2^{p} \alpha_{p}\right)^{5 / 4} T_{r}}\right)^{12 n^{2}-5}\right]^{\frac{4}{(5 p+4)\left(-20 n^{2}+5\right)}}
\end{aligned}
$$

When $K<0$, the upper limit for the mass is obtained from above equation

$$
m<\left(\frac{\left(2^{p} \alpha_{p}\right)^{5 / 2}}{1.3 \times 10^{30} a^{2 / \beta}}\right)^{\frac{4 \beta}{(5 p+4) \beta+8}}
$$


FIG. 1: We plot the parameter $m^{2}$ in term of dissipation parameter $\Gamma_{0}$ where $T=T_{r}=2.24 \times$ $10^{16} \mathrm{GeV}, K_{*}=0.002 M p c^{-1}$ and $\kappa=1$

This upper limit is also obtained from Planck data [44, 45]

$$
m<\left(\frac{\left(2^{p} \alpha_{p}\right)^{5 / 2}}{1.3 \times 10^{30} a^{\prime 2 / \beta}}\right)^{\frac{4 \beta}{(5 p+4) \beta+8}}
$$

where

$$
a=\left[\left(\frac{2.7 \times 10^{-10}}{\kappa^{3 / 4} T_{r}}\right)^{4 n^{2}-5}\left(\frac{465 \kappa^{5 / 4} \operatorname{coth}(k / 2 T)}{\left(2^{p} \alpha_{p}\right)^{5 / 4} T_{r}}\right)^{12 n^{2}-5}\right]^{\frac{4}{(5 p+4)\left(-20 n^{2}+5\right)}}
$$

\section{CONCLUSION}

In this article we have studied warm-polytropic inflationary model. We have obtained explicit expressions for the tensor-scalar ratio $R$, scalar spectrum index $n_{s}$ and its running $\alpha_{s}$, in slow-roll approximation. We have developed our specific model by a chaotic potential, for two different cases of the dissipative parameter $\Gamma$ (where $\Gamma \gg 3 H$ ). $1-\Gamma$ is a constant parameter and $2-\Gamma$ is a function of scalar field. In these cases we have found exact solution for the scalar field and Hubble parameter. We also have obtained a relation between scalar field and radiation field energy densities. By using WMAP7 and forthcoming Planck observation 
data we have constrained the mass of inflaton and the chaotic potential.

[1] A. Guth, Phys. Rev. D. 23, 347 (1981).

[2] A. Albrecht and P. J. Steinhardt, Phys. Rev. Lett. 48, 1220 (1982).

[3] D. N. Spergel, "First year Wilkinson microwave anisotropy probe (WMAP) observations: Determination of cosmological parameters," Astrophys. J. Suppl. 148, 175, (2003).

[4] H.V. Peiris, "First year wilkinson microwave anisotropy probe (WMAP) observations: Implications for inflation," Astrophys. J. Suppl. 148, 213, (2003).

[5] A. Berera, "Warm inflation," Phys. Rev. Lett. 75, 3218, (1995).

[6] A. Berera, "Interpolating the stage of exponential expansion in the early universe: A possible alternative with no reheating," Phys. Rev. D 55, 3346, (1997).

[7] L. M. H. Hall, I. G. Moss and A. Berera, Phys.Rev.D 69, 083525 (2004).

[8] I.G. Moss, Phys.Lett.B 154, 120 (1985).

[9] A. Berera and L.Z. Fang, Phys.Rev.Lett. 741912 (1995).

[10] A. Berera, Nucl.Phys B 585, 666 (2000).

[11] U. Mukhopadhyay and S. Ray, Mod. Phys. Lett. A 23, 3198, (2008).

[12] J. Christensen-Dalsgard, Lecture Notes on Stellar Structure and Evolution, 6th edn. (Aarhus University Press, Aarhus, 2004).

[13] R N Tiwari, J R Rao and R R Kanakamedala Phys. Rev. D 341205 (1986).

[14] S. Ray Astrophys. Space Sci. 280345 (2002).

[15] J. D. Murray, Mathematical Biology (Springer, Berlin, 1991).

[16] C. Tsallis, Introduction to Nonextensive Statistical Mechanics (Springer, 2009).

[17] S. Chandrasekhar, An Introduction to the Study of Stellar Structure (Dover, 1958).

[18] S. Shapiro, S. A. Teukolsky, Black Holes, White Dwarfs, and Neutron Stars (Wiley, 1983).

[19] P.-H. Chavanis, "A simple model of universe with a polytropic equation of state," arXiv:1208.1192 [astro-ph.CO].

[20] P.-H. Chavanis, "Models of universe with a polytropic equation of state: III. The phantom universe," arXiv:1208.1185 [astro-ph.CO].

[21] P.-H. Chavanis, "Models of universe with a polytropic equation of state: II. The late universe," arXiv:1208.0801 [astro-ph.CO]. 
[22] O. Bertolami and V. Duvvuri, Phys. Lett. B 640, 121 (2006).

[23] R. Herrera, Phys. Lett. B664, 149, (2008).

[24] S. del Campo, R. Herrera, Phys. Lett. B665, 100, (2008).

[25] M. Bouhmadi-Lopez, P.F. Gonzalez-Diaz, P. Martin-Moruno, arXiv: 0707.2390 [gr-qc].

[26] Y.S. Myung, Phys. Lett. B 652 (2007) 223, arXiv: 0706.3757 [gr-qc].

[27] L. Randall, R. Sundrum, Phys. Rev. Lett. 83 (1999) 4690.

[28] T. Shiromizu, K. Maeda, M. Sasaki, Phys. Rev. D 62 (2000) 024012.

[29] D. Dvali, G. Gabadadze, M. Porrati, Phys. Lett. B 485 (2000) 208

[30] K. Freese, M. Lewis, Phys. Lett. B 540 (2002) 1.

[31] R. Maartens, Lect. Notes Phys. 653 (2004) 213.

[32] A. Lue, Phys. Rep. 423 (2006) 1.

[33] A. Liddle, D. Lyth, Cosmological Inflation and Large-Scale Structure, Cambridge Univ. Press, 2000 .

[34] J. Linsey, A. Liddle, E. Kolb, E. Copeland, Rev. Mod. Phys. 69 (1997) 373.

[35] B. Bassett, S. Tsujikawa, D. Wands, Rev. Mod. Phys. 78 (2005) 537.

[36] A. Taylor and A. Berera, Phys. Rev. D 69, 083517 (2000).

[37] D. Larson, et al. ApJ, 193,16 (2011).

[38] K. Bhattacharya, S. Mohanty and A. Nautiyal, Phys. Rev. Lett.97, 251301, (2006).

[39] E. Komatsu et. al, Astrophys. J. Suppl. 192, 16, (2011).

[40] W. Lee and L. Z. Fang, Phys. Rev. D 59, 083503 (1999)

[41] H. Oliveira, Phys. Lett. B 526, 1 (2002).

[42] H. Oliveira and S. Joras, Phys. Rev. D 64, 063513 (2001).

[43] M. A. Cid, S. del Campo and R. Herrera, JCAP 0710, 005 (2007) [arXiv:0710.3148 [astro-ph]].

[44] P. Ade et al. (Planck Collaboration), Astron.Astrophys. 536, 16464 (2011).

[45] P. Ade et al. astro-ph/0604069; URL http://www.rssd.esa.int/index.php?project=Planck. 